\title{
Improving patient outcomes through innovative, community- based HIV treatment strategies
}

Project SOAR

Follow this and additional works at: https://knowledgecommons.popcouncil.org/departments_sbsr-hiv

Part of the Health Services Research Commons How does access to this work benefit you? Let us know!

\section{Recommended Citation}

Project SOAR. 2021. "Improving patient outcomes through innovative, community-based HIV treatment strategies," Learnings from Project SOAR, Synthesis Brief. Washington, DC: Population Council. 


\section{Improving Patient Outcomes through Innovative, Community-Based HIV Treatment Strategies}

\section{Learnings from Project SOAR}

Despite the availability of life-saving treatment for HIV, several barriers prevent patient access to high-quality services. HIV program managers and policymakers have shown increasing interest in understanding how best to deliver HIV treatment to different populations and maximize health system resources at facility and community levels.

Project SOAR recognizes the key role that treatment services play in achieving UNAIDS' $90-90-90$ goals for controlling the HIV epidemic through testing, treatment, and viral suppression by 2020 . Drawing on its extensive portfolio, Project SOAR has contributed to the evidence base and catalyzed research use by assessing innovative strategies for antiretroviral treatment (ART) delivery which support different populations to remain in care, reach viral suppression, and achieve positive health outcomes.

This brief synthesizes results from 15 studies across 9 countries (see map on next page for additional detail) that examined:

- Innovations in ART guidelines, including Test and Start and multi-month dispensing

- Facility-based psychosocial support models for adults, children, and caregivers of people living with HIV

- Multiple community platforms and resources to deliver ART and/or support people living with HIV (PLHIV) on ART

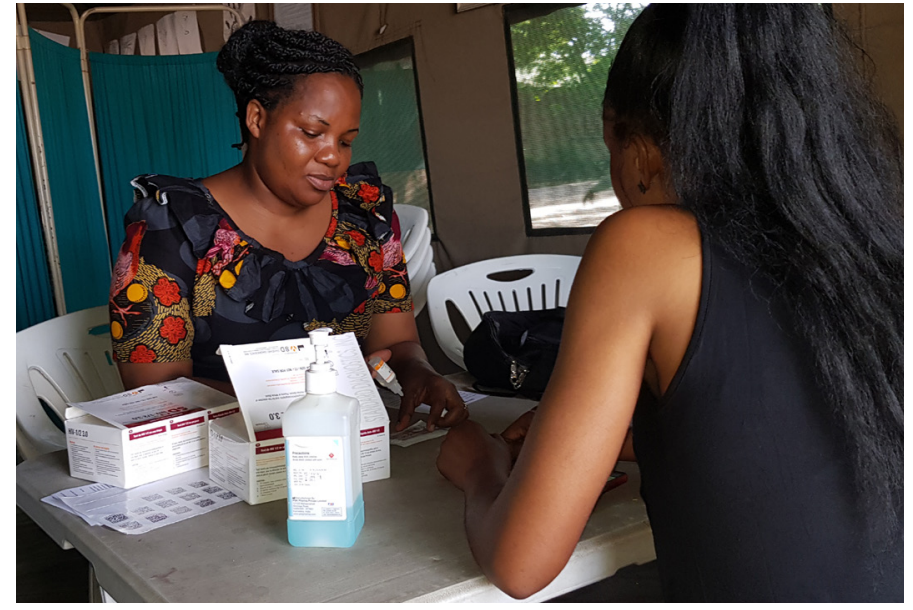

KEY RECOMMENDATIONS

1. Continue Test and Start and multi-month dispensing, ensuring adequate training of health providers and availability of supplies to improve viral load testing. While Test and Start can improve treatment initiation, additional measures are needed to improve retention and viral load testing, particularly for key populations at higher risk of HIV.

2. Provide psychosocial support through community- and facility-based models to improve patient outcomes. Facility-based support groups for children and adolescents were positively associated with well-being, adherence, and viral suppression.

3. Expand the use of community-based platforms to promote access to highquality care, especially for marginalized populations. Use of community-based models, using community health workers and drop-in centers, strengthened HIV service delivery, client satisfaction, and antiretroviral treatment initiation among women, children, and female sex workers. 


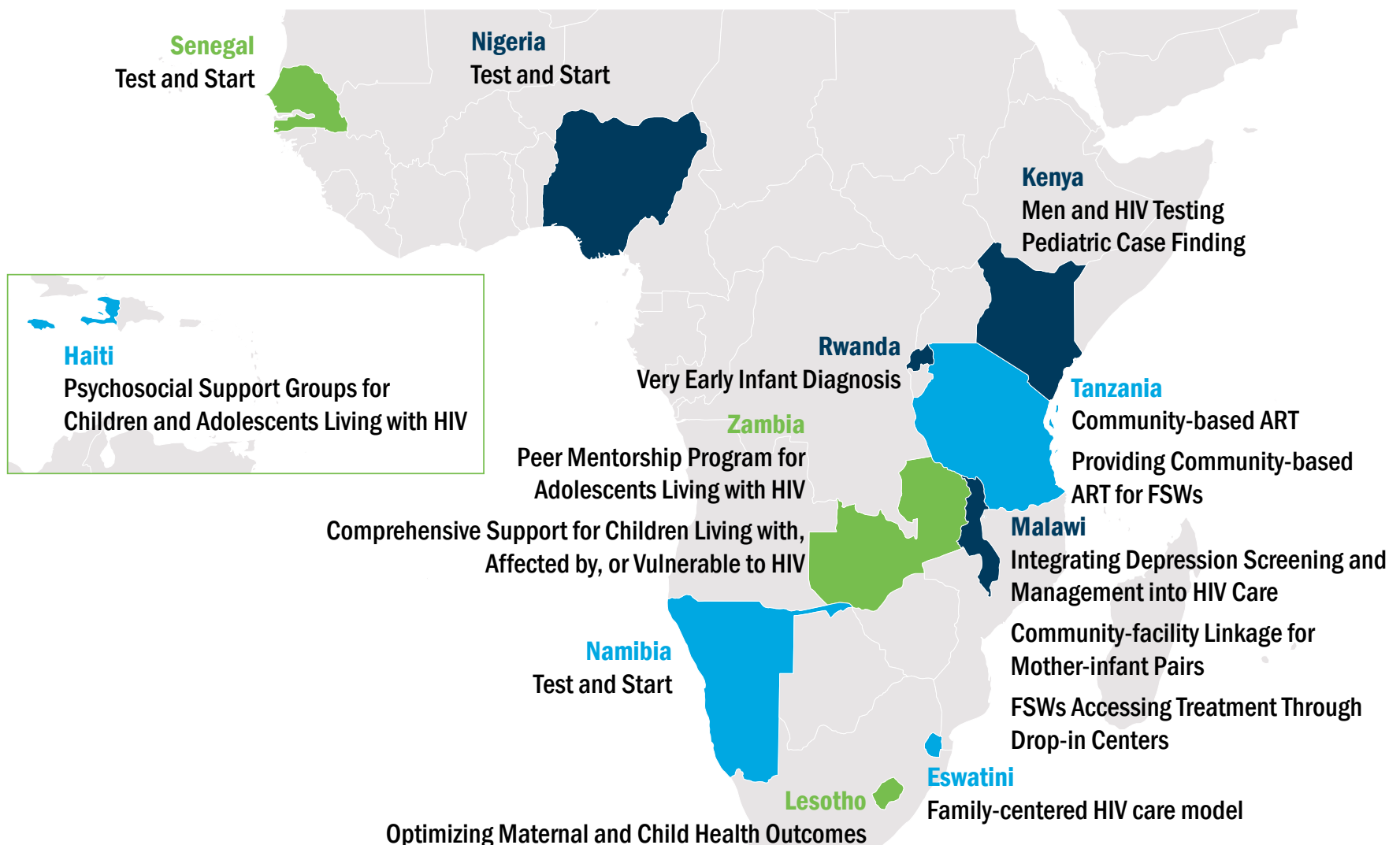

Through Use of Multidisciplinary Teams

\section{ABOUT}

Project SOAR (Supporting Operational AIDS Research), was a 6-year USAID-funded implementation science initiative with over 70 activities in 21 countries. The Population Council led Project SOAR in collaboration with Avenir Health, Elizabeth Glaser Pediatric AIDS Foundation (EGPAF), Johns Hopkins University, Palladium, and The University of North Carolina at Chapel Hill.

\section{Research Utilization}

Project SOAR implemented a systematic and proactive research utilization (RU) approach to advance translation of research results into evidence-informed practice. This $\mathrm{RU}$ approach supported inclusion of end data users in the research process from inception to dissemination and fostered data-driven policy and program change.

\section{Series}

"Learnings from Project SOAR" seeks to highlight key results, recommendations, and examples of research impact across this 6-year, 21-country initiative.

The series includes five thematic briefs focused on biomedical prevention interventions, HIV testing services, HIV treatment strategies, stigma, and research utilization and capacity strengthening.

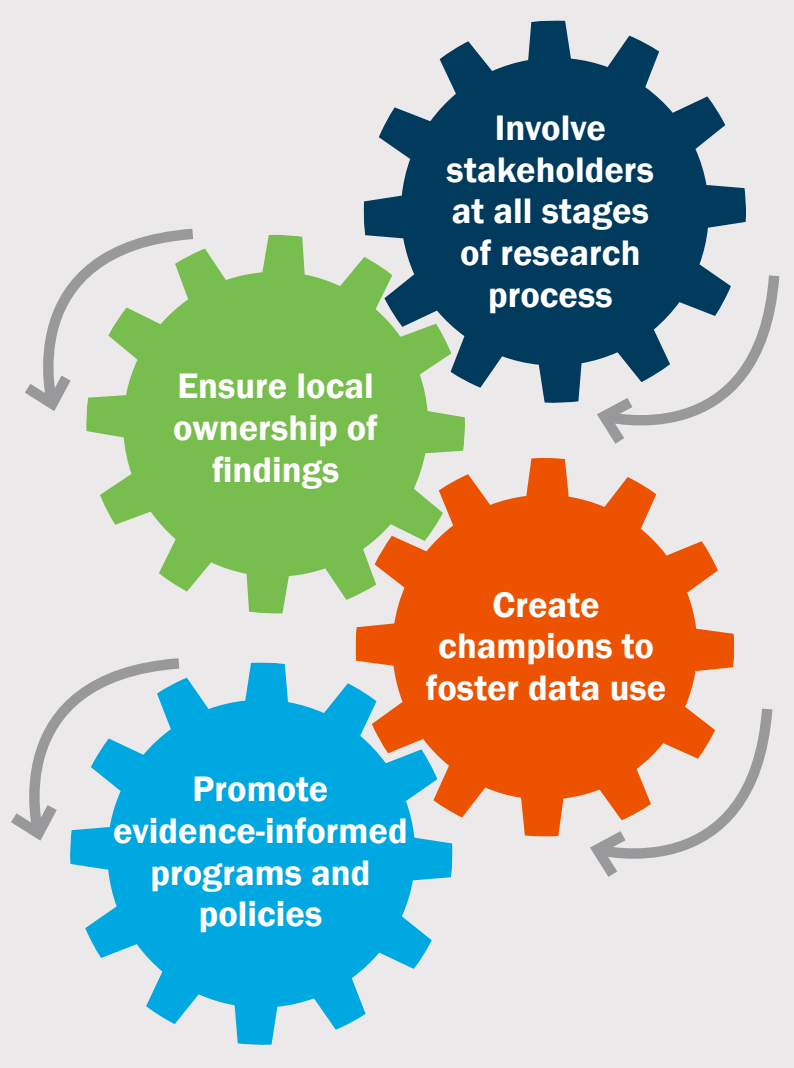

2 | Improving patient outcomes through innovative, community-based HIV treatment strategies 
INNOVATIONS IN ART GUIDELINES: TEST AND START AND MULTIMONTH DISPENSING

Overview: Based on evidence demonstrating that early initiation of ART leads to better health outcomes, the WHO-recommended Test and Start model puts patients on ART immediately after an HIV-positive diagnosis. Multi-month dispensing (MMD) of ART has also emerged as a community-oriented and time saving approach for stable patients and clinicians. SOAR examined the impact of these ART distribution models on treatment outcomes, including initiation and retention in care, in Namibia, Nigeria, Senegal, and Tanzania.

Results summary: These studies found that while Test and Start may improve treatment initiation, additional measures are needed to improve retention and viral load testing, particularly for key populations. MMD appears to be a safe alternative to single month dosing and led to improved treatment outcomes.

\section{Key findings}

In Namibia, national roll out of Test and Start in rural district hospitals and community-based health facilities did not compromise key treatment outcomes among clients. Time to ART initiation decreased significantly and more than 90 percent of patients were retained in care after 12 months.

Namibia began implementing the Treat All (Test and Start) guidelines in April 2017, which stipulated that anyone who tests positive for HIV (regardless of CD4 count) begins ART as soon as possible. SOAR researchers abstracted information from client records in Northern Namibian district hospitals and community clinics to examine changes in key treatment outcomes before implementation of the new guidelines, and at 6 and 12 months after Treat All began. Research results showed that clients' median time to initiation decreased significantly post-Treat
Figure 1 Test and Start in Namibia

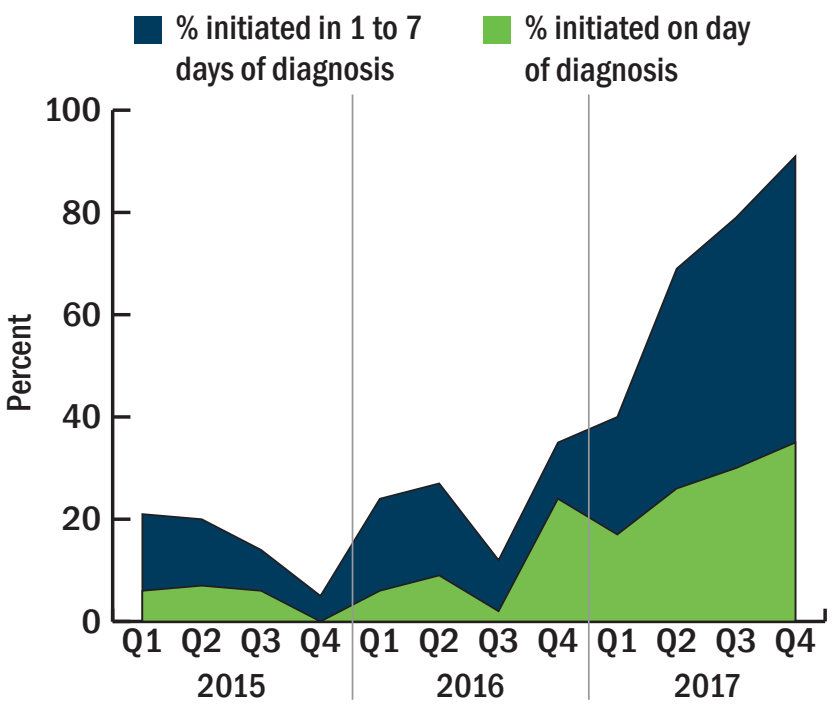

All, and study facilities reached two critical milestones: more than $\mathbf{9 0}$ percent of ART patients were retained in care 12 months after ART initiation, and $\mathbf{9 0}$ percent of clients who received a viral load test achieved viral suppression. However, while viral load testing improved over time, the proportion of clients being tested remained low. Additionally, most patients lacked basic knowledge of viral load testing. ${ }^{1}$

In Senegal and Nigeria, ART initiation and retention proved more challenging among clients exposed to two unique Test and Start strategies, many of whom were female sex workers (FSWs) and men who have sex with men (MSM).

\section{Viral load testing was low across all study} sites; however, in Namibia and Nigeria, at least 90 percent of those tested achieved viral suppression.

In Nigeria, SOAR researchers assessed the performance of two community-based Test and Start service delivery models for key populations (MSM and FSWs). The models comprised HIV prevention drop-in centers (DICs) supported by a separate implementing partner that provided ART services, and one-stopshop (OSS) clinics operated by a full-time clinical team offering comprehensive care and treatment services all by one organization. Participants were recruited via DIC-organized community outreach and from OSS walk-in clients, and those who tested positive were 
offered same-day ART. Of the 605 recruited clients, 71 percent were successfully linked to treatment, well below the $\mathbf{9 0}$ percent UNAIDS goal, and this did not differ by model. ART initiation was significantly higher among MSM compared to FSWs. Retention at one year was poor, with just 56 percent of clients who initiated ART remaining in treatment; however, retention was higher in the OSS model than in the DIC model and higher among MSM compared to FSWs. Overall, viral load testing was low; 71 percent of those who initiated treatment received at least one viral load test. Yet, among these clients, viral suppression was high at $\mathbf{9 6}$ percent. $^{2}$ While the OSS model performed slightly better than the DIC model (73\% linkage and $48 \%$ retention versus $68 \%$ linkage and $63 \%$ retention, respectively), there is still a need to improve treatment outcomes in both models as overall there was still extremely low levels of linkage and retention.

In Senegal, SOAR researchers conducted a randomized controlled trial to compare individual case management to the standard of care (SOC) as part of the country's roll-out of Test and Start. The case management intervention was a multi-step process to coordinate care and provide a family-like support system for PLHIV. Between one-third and one-half of the 573 participants assigned to either the intervention or SOC were members of key populations (FSWs and MSM). The proportion of all participants who were virally suppressed at $\mathbf{1 2}$ months ranged from $\mathbf{7 0}$ percent to $\mathbf{7 7}$ percent, below the target of 90 percent. The study found no difference in viral suppression between clients in the intervention group versus the control group, and loss to follow-up was high. About half of participants were retained by the end of the study. ${ }^{3}$

\section{In Tanzania, MMD appeared to be a safe} alternative to single month dosing. Those receiving at least one 3-month ART prescription had better treatment outcomes than those who did not.

Using patient-level clinical monitoring data before and after introduction of Tanzania's MMD program for stable ART clients, SOAR examined MMD's contributions to treatment outcomes. Specifically, the study compared retention, adherence, viral load, and mortality between two comparable cohorts:
ART clients receiving 3-month MMD and those receiving only monthly prescriptions. Both male and female patients who received at least one 3-MMD were more likely to stay on treatment and have undetectable or suppressed viral load results after 12 months. Moreover, MMD did not lead to safety issues or adversely affect mortality, emerging as a safe alternative to single month dosing. ${ }^{4}$

\section{FACILITY-BASED PSYCHOSOCIAL SUPPORT TO ADULTS, CHILDREN, AND CAREGIVERS}

Overview: Evidence suggests that poor mental

health and lack of social support remain

barriers to consistent HIV care engagement

and long-term viral suppression. Project

SOAR examined the effects of facility-based

psychosocial support models for children and

adults living with HIV in Eswatini, Haiti, Malawi,

and Zambia.

Results summary: Overall, these support

activities were found to be feasible and

acceptable, with the potential to improve

wellbeing outcomes and viral suppression.

However, more intensive support may be

needed for those with depression.

\section{Key findings}

In Haiti, participation in facility-based, support groups for children and adolescents living with HIV was positively associated with children's well-being, adherence, and viral suppression.

In Haiti, Kids Clubs provide hospital-linked psychosocial support groups for young people living with HIV ages nine and older. Project SOAR conducted a retrospective study of the program and found that it had a positive effect on participants, including clinical outcomes, and had scaled over time to reach a larger population. The number of health facilities hosting clubs grew from 6 to 24, and enrollment increased nearly tenfold from 2014 to 2018. Importantly, club members, their caregivers, 
and program implementers reported the clubs had helped members keep their clinic appointments and about three-fourths of club participants reported consistent treatment use at their most recent clinic visit. However, a total of 64 percent of Kids Club members with a recent viral load assessment were virally suppressed, substantially below the 90 percent threshold. The level of club attendance was associated with ART adherence and viral load suppression. ${ }^{5}$

\section{In Zambia, viral suppression increased and} self-reported adherence gaps and internalized stigma decreased among youth participating in a facility-based, peer support model.

In Zambia, SOAR researchers conducted a randomized controlled trial in four HIV clinics (pediatric and adult facilities) to test a peer mentorship model for youth living with HIV (15-24 years of age). Participants were randomly assigned in each clinic to either the intervention arm (Project YES!) consisting of monthly one-on-one and small group sessions with a youth peer mentor (YPM) and optional caregiver support groups, or the usual care arm. While viral suppression increased significantly in both study arms at six months, in a stratified analysis, participants in the YES! arm in one of the sites (a pediatric hospital) experienced an increase in viral suppression by a factor of 4.7 relative to the comparison arm. Moreover, reported adherence gaps (not taking ART for $\mathbf{4 8}$ or more consecutive hours) and internalized stigma significantly decreased among youth in the intervention arm across facilities. YPMs noted how Project YES! changed their clients' lives and their own, resulting in powerful personal transformations in HIV-related health behaviors, conceptions of self, and plans for the future. Many expressed seeing themselves now as community leaders"ambassadors," "game changers," and "not just alone in this world."6

In Eswatini, a family care model demonstrated no impact on retention, reported adherence, or viral suppression among participating children. But caregivers and health workers cited benefits of the model such as facilitating disclosure and support among family members.
SOAR researchers tested a family centered care model in Eswatini whereby HIV-positive children and adolescents ( $<19$ years) were provided treatment services directly involving at least one family member. Participants saw the same provider at each visit and medications for family members could be collected by the family member. Overall, viral suppression improved among children and adolescents in both study arms from 78 percent at enrollment to 90 percent at 12 months and 87 percent for those followed to 18 months. Similarly, there were no significant differences by group in reported missed clinic visits, interruption of ART, reported adherence, or disclosure of HIV status. The family-centered care model program, however, was well-liked by both caregivers and health care workers. Caregivers thought the model encouraged men to take a proactive role in supporting women and children and reduced HIV-related stigma within the family. ${ }^{7}$

\section{In Malawi, integration of depression screen-} ing and management into HIV care was feasible and acceptable, but the program did not appear to improve HIV or mental health outcomes.

SOAR researchers evaluated the implementation of integrating depression screening and management into HIV care at two public HIV clinics in Malawi. All patients starting ART and identified as having mild, moderate, or severe depressive symptoms (PHQ-9 score $\geq 5$ ) were followed for six months after ART initiation to track HIV appointment attendance, viral load, and depressive symptom outcomes. Depression screening and treatment initiation proved highly feasible to implement. Among patients initiating ART, 96 percent completed the depression screening process. Of those identified with mild depression and moderate to severe depression, 87 percent initiated counseling and 96 percent initiated an antidepressant, respectively. Sustaining depression treatment proved challenging, in part because many patients missed follow-up ART appointments or were quickly lost to care. Overall, the program did not appear to improve HIV or mental health outcomes. ${ }^{8}$ 


\section{COMMUNITY PLATFORMS AND RESOURCES TO DELIVER ART AND/OR SUPPORT PLHIV ON ART}

Overview: Many marginalized populations struggle to access health facilities and see community-based health care services as more accessible and welcoming. Project SOAR assessed the outcomes of a variety of community-facility linkage models across diverse contexts in Lesotho, Malawi, Tanzania, and Zambia.

Results summary: Community-based

approaches, featuring community health workers and drop-in centers, demonstrated promising results for mothers and infants, adult patients, and female sex workers and their partners. These approaches resulted in improvements in HIV service delivery, client satisfaction, and ART initiation across these key populations.

\section{Key findings}

In Malawil, three widely used community-facility linkage (CFL) models all contributed to improved HIV service delivery for mothers and infants.

To improve health outcomes among mother-infant pairs (MIPs), three CFL models have been widely implemented in Malawi as part of its national PMTCT program: 1) mentor mothers; 2) expert clients; and 3) community health workers. SOAR researchers examined the relative impact of these models and the standard of care based on routinely collected data for MIPs. All CFL models reached approximately 95 percent of MIPs. Overall, CFL models contributed to strengthening HIV service delivery for MIPs by providing additional human resources, improving service reach and function, building long-term relationships between clients and providers, and better linking facility with community health services. Mothers living with HIV participating in CFL models spent more time in care, on ART, and virologically suppressed when: 1) CFL providers were individually assigned 30 or fewer MIPs to follow and 2) CFL providers made more frequent routine home visits to clients (i.e., $>2$ per quarter). ${ }^{9}$

In Lesotho, both HIV-positive and HIVnegative women who received services from patient-centered, multidisciplinary integrated management teams had better HIV outcomes and were more satisfied with services than those in the control group.

SOAR researchers in Lesotho compared the effectiveness of the IMPROVE intervention among a cohort of HIV-positive and HIV-negative pregnant/ postpartum women and their infants and a similar cohort of women receiving routine care. The intervention included three key approaches: 1) multidisciplinary management teams of facility- and community-based health care and lay workers, 2) skills-building training and job aids for health workers, and 3 ) increased early community-based counseling and support for patients. The prospective, mixedmethods study demonstrated improved clinical outcomes, such as adherence by pill count and undetectable viral load, among HIV-positive women in the intervention arm compared to women in the control arm, but not all changes were sustained over a 24-month period. HIV-negative women in the intervention arm were retained in care longer and were more likely to be retested for HIV prior to delivery. Women in the intervention arm were more satisfied with the health services they received, while health care workers felt the IMPROVE intervention was feasible and acceptable, and improved their communication with patients and delivery of services. ${ }^{10}$

In Zambia, young people living with HIV had good HIV and well-being outcomes in communities with integrated service provision and comparison communities, but there was no difference between these groups.

The Zambia Family (ZAMFAM) Program focused on strengthening comprehensive, integrated service delivery and providing support to children living with, affected by, or vulnerable to HIV in Zambia. SOAR researchers conducted a prospective cohort study of young people living with HIV and their primary

6 I Improving patient outcomes through innovative, community-based HIV treatment strategies 
caregivers/guardians to determine the effect of ZAMFAM activities on the well-being of young people living with HIV and their households. At 12 months, the study detected no difference in HIV and wellbeing indicators between young people living with HIV in communities where integrated services were provided compared to youth in comparison communities. More than 90 percent of young people living with HIV in ZAMFAM and non-ZAMFAM areas continued to adhere to treatment and remained in care, and self-reported viral load testing increased in both areas. Access to psychosocial support increased among older young people living with HIV in both areas; however, many children still cannot access these services. Food insecurity, financial instability, sickness (non-HIV related), and school attendance remained major problems among young people living with HIV and their households. ${ }^{11}$

\section{In Tanzania, PLHIV accessing ART through} a community-based model were significantly more likely to be retained in care at 12 months and report lower levels of internalized stigma compared to the control group.

PLHIV accessing ART through a community-based model in Tanzania were significantly more likely to be retained in care at 12 months. There were no significant differences in retention between men and women. Internalized stigma significantly reduced in the intervention group from 6 to 12 months whereas it increased in the control group. The study found no difference in adherence or viral suppression. Clients, clinicians, and community-based health agents reported high levels of satisfaction with the intervention. ${ }^{12}$

In Tanzania, FSWs who received HIV treatment through a community-based service delivery model were more likely to initiate $A R T$, remain in care at 6 and 12 months, and be satisfied with services than those who accessed care through health facilities. The program was also successful in recruiting FSW's partners to get tested, and if positive, initiate treatment.

Recognizing the many challenges FSWs face in accessing HIV care and treatment services, SOAR researchers conducted a quasi-experimental prospective study in Tanzania to evaluate a community-based model to deliver ART via mobile HIV testing/counseling services and home visits. ART initiation was significantly higher in the intervention arm compared to the comparison arm at six months. Retention was significantly higher in the intervention arm compared to the comparison arm at both 6 months and 12 months. Viral suppression among those who initiated ART was fairly high at 6 and 12 months, with no differences by arm. Satisfaction with ART services, however, was higher among FSWs receiving the community-based ART services. ${ }^{13}$ Almost one year into the study, it was expanded to offer the same HIV testing and treatment services to their regular sex partners. SOAR found that partner testing and treatment initiation through the community-based ART program was feasible and acceptable, facilitating treatment initiation. Partners felt they received more patient-centered care than they would from regular clinics and experienced less stigma. ${ }^{14}$

\section{In Malawi, FSWs who accessed ART services through DICs had higher retention rates at 12 months than those who only used health facil- ities or a combination of DICs and facilities. FSWs found DICs easier to access and more private than public facilities.}

In another study focused on FSWs, SOAR researchers assessed the preference for and early effectiveness of a community-based ART program in Malawi. FSW participants were given the option to initiate treatment through either public facilities or DICs located near hotspots and could change modalities after six months. Retention at 12 months was highest among exclusive DIC clients compared to facilitybased or mixed-site clients. Although viral load tests were not frequently documented, clients who had ever collected ART medication through a DIC were nearly twice as likely to have documented viral load results compared to those who exclusively collected their ART medication from health facilities. After one year, nearly two-thirds had accessed DIC-based ART services since registering in care, citing that DICs were easier to access, more private, and staffed by caring providers. ${ }^{15}$ 


\section{RESEARCH IMPACT SPOTLIGHT}

\section{Tanzania study informs national policy change on community-based ART delivery}

Informed by Project SOAR findings demonstrating the value of community-based delivery of ART to FSWs, the Government of Tanzania changed national guidance to allow for community-based HIV treatment to better meet the needs of marginalized populations. Read more here.

\section{REFERENCES}

1. Vu, L. et al. 2020. "Assessing the effects of the implementation of the WHO "Treat All" guidelines on treatment outcomes, service quality, and cost in Namibia," Project SOAR Final Report. Washington, D.C.: Population Council

2. Eluwa, G. et al. 2021. "Assessment of HIV testing misclassifications, and effectiveness of community-based HIV service delivery models among key populations in Nigeria," Project SOAR Final Report. Washington, D.C.: Population Council

3. Baral, S. et al. 2021. "Leveraging adaptive implementation strategies to achieve universal coverage of antiretroviral treatment in Senegal," Project SOAR Final Report. Washington, D.C.: Population Council

4. Apicella, L. et al. 2021. "Assessment of multi-month dispensing for anti-retroviral treatment of HIV in Tanzania: policy, outcomes, and costing," Project SOAR Final Report. Washington, D.C.: Population Council

5. Settergren, S. et al. 2020. "Support clubs for Haitian children and youth living with HIV: A case study," Project SOAR Final Report. Washington, D.C.: Population Council.

6. Denison, J. et al. 2020. "Project YES! Youth engaging for success: A Randomized Controlled Trial testing a youth peer mentoring approach among HIV-positive adolescents and young adults in Ndola, Zambia," Project SOAR Final Report. Washington, D.C.: Population Council

7. Chouraya, C. et al. 2020. "Piloting and evaluating familycentered care in Eswatini," Project SOAR Final Report. Washington, D.C.: Population Council

8. Pence, B. et al. 2020. "Integrating depression management into HIV care in Lilongwe, Malawi: feasibility and impact," Project SOAR Final Report. Washington, D.C.: Population Council.

9. Herce, M. et al. 2020. “Improving PMTCT outcomes for mother-infant pairs through community-facility linkage: results from a mixed methods study in Malawi," Project SOAR Final Report. Washington, D.C.: Population Council
10. Tiam, A. et al. 2020. "Optimizing maternal and child health outcomes through use of multidisciplinary "IMPROVE" teams in Lesotho," Project SOAR Final Report. Washington, D.C.: Population Council

11. Mbizvo, M. et al. 2018. "Benchmark assessment of orphaned and vulnerable children in areas of the Zambia Family (ZAMFAM) Project," Project SOAR Final Report. Washington, D.C.: Population Council

12. Mkopi, Abdallah et al. 2021. "Impact of a communitybased support intervention on antiretroviral therapy retention in Tanzania," Project SOAR Final Report. Washington, D.C.: Population Council

13. Vu, L et al. 2020. “Community-based HIV treatment service delivery model for female sex workers in Tanzania: evaluation findings," Project SOAR Final Report. Washington, D.C.: Population Council.

14. Apicella, L. et al. 2020. "Community-based delivery of antiretroviral therapy (ART) for partners and children of female sex workers in Tanzania: Feasibility and acceptability assessment," Project SOAR Brief. Washington, D.C.: Population Council

15. Vu, L. et al. 2020. "Assessment of community-based ART service model linking female sex workers to HIV care and treatment in Blantyre and Mangochi, Malawi," Project SOAR Final Report. Washington, D.C.: Population Council.

Suggested citation: Project SOAR. 2021. "Improving patient outcomes through innovative, community-based HIV treatment strategies," Learnings from Project SOAR Synthesis Brief. Washington, D.C.: Population Council.

For more information, please visit Project SOAR.
Project SOAR was a six-year (September 2014-January 2021) cooperative agreement funded by the United States President's Emergency Plan for AIDS Relief and the U. S. Agency for International Development (Agreement No. AID-OAA-A-14-00060). The contents of this brief are the sole responsibility of Project SOAR and Population Council and do not necessarily reflect the views of PEPFAR, USAID, or the United States Government.

Population Council led the Project SOAR consortium in collaboration with Avenir Health, Elizabeth Glaser Pediatric AIDS Foundation, the Johns Hopkins University, Palladium, and The University of North Carolina at Chapel Hill.
Project SOAR/Population Council

4301 Connecticut Avenue, NW, Suite 280

Washington, DC 20008

Tel: +12022379400

e-mail: ProjectSOAR@popcouncil.org projsoar.org

@Population Council, April 2021

Cover photo credit: CSK Research Solutions 